\title{
Avaliação do Comprimento do Colo Uterino nas Posições Ortostática e Decúbito Horizontal nas Gestações Gemelares
}

\author{
Uterine Cervical Length Evaluation in the Standing and Recumbent \\ Positions in Twin Pregnancies \\ Tatiana Bernáth, Maria de Lourdes Brizot, Adolfo Wenjaw Liao, Luciana Cury, \\ Jorge Demétrio Banduki, Marcelo Zugaib
}

\begin{abstract}
RESUM0
Objetivo: avaliação ultra-sonográfica e comparação da medida do comprimento do colo uterino nas gestações gemelares com as pacientes nas posições de decúbito dorsal horizontal (DDH) e ortostática.

Métodos: 50 gestações gemelares foram submetidas a avaliações ultra-sonográficas para medida do comprimento do colo uterino no periodo de maio de 1999 a dezembro de 2000. Os exames foram realizados pela via transvaginal com periodicidade de 4 semanas totalizando 136 avaliações. A cérvice uterina foi avaliada, segundo técnica normatizada, com a paciente nas posições de decúbito dorsal horizontal e ortostática.

Resultados: as medidas do colo uterino nas posições $D D H$ e ortostática na primeira avaliação apresentaram correlação inversa com a idade gestacional (DDH: $r=-0,60 ; p<0,001$; ortostático: $r=-0,46 ; p=0,008)$. A média da medida do colo uterino em $D D H$ foi de 35, $2 \mathrm{~mm}(D P=9,9 \mathrm{~mm}), e$ $33,4 \mathrm{~mm}(D P=9,5 \mathrm{~mm})$ na posição ortostática $(p=0,06)$. Quando a diferença entre as medidas obtidas nas posiões ortostática e DDH era expressa como percentual da medida na posição $D D H$, não houve correlação significativa com a idade gestacional $(p=0,07)$ e a média das diferenças percentuais foi de $-2,9 \%(p=0,3)$. Comparando-se todas as avaliações, houve correlação significativa entre as medidas do colo nas posições DDH e ortostática $(r=0,79$; $p<0,001)$. A média da medida do colo uterino em $D D H$ foi de 33,5 $\mathrm{mm}(D P=10,8 \mathrm{~mm})$ e $31,8 \mathrm{~mm}(D P=9,6 \mathrm{~mm})$ na posição ortostática $(p=0,003)$. A diferença percentual não apresentou correlação significativa com a idade gestacional da avaliação $(p=0,10)$ e a média das diferenças percentuais foi de $-3,0 \%(p=0,10)$.

Conclusão: as medidas do comprimento do colo uterino variam inversamente com a idade gestacional. Apesar de haver diferenças significativas entre as medidas absolutas em DDHe ortostática, quando essa diferença é expressa como percentual da medida do colo em decúbito, ela não foi significativa. Portanto, a avaliação cervical nas posições de decúbito e ortostática fornecem informações semelhantes.
\end{abstract}

PALAVRAS-CHAVE: Prematuridade. Gemelaridade. Colo uterino. Gravidez normal.

\section{Introdução}

Nas últimas décadas tem-se observado au-

Departamento de Ginecologia e Obstetrícia do Hospital das Clínicas da Faculdade de Medicina da Universidade de São Paulo

Correspondência:

Maria de Lourdes Brizot

Departamento de Ginecologia e Obstetrícia

Av. Dr. Enéas de Carvalho Aguiar, 255- $10^{\circ}$ andar - Cerqueira César

05403-000 - São Paulo - SP

e-mail: mlbrizot@uol.com.br mento na incidência de gestações gemelares devido à introdução e difusão das técnicas de reprodução assistida e aumento da idade média mater$\mathrm{na}^{1}$. Gestações gemelares, quando comparadas com gestações únicas, são mais freqüentemente associadas a complicações e, dentre estas, vale ressaltar o parto pré-termo ${ }^{2}$.

Tradicionalmente, a avaliação do colo uterino é realizada por meio de exame digital. Entretanto, o exame ultra-sonográfico transvaginal permite avaliar sua porção supravaginal e estimar a real medida de seu comprimento. É técni- 
ca de fácil execução e boa reprodutibilidade ${ }^{3,4}$.

A medida do comprimento do colo uterino pela ultra-sonografia transvaginal tem mostrado ser um bom marcador para predição de parto prematuro tanto nas gestações únicas como nas gemelares. Iams et al. ${ }^{5}$ em estudo multicêntrico e prospectivo, avaliaram 2.915 gestações únicas em 2 períodos. A primeira avaliação foi feita na $24^{\mathrm{a}}$ e a segunda na $28^{\text {a }}$ semana de gestação. Os seus resultados mostraram que o risco para parto prematuro aumentava à medida que o comprimento do colo uterino diminuía durante a gestação. Goldenberg et al. ${ }^{6}$ avaliaram 147 gestações gemelares e concluíram que o melhor preditor de parto pré-termo foi a medida do comprimento cervical obtida durante a $24^{\text {a }}$ semana; quando esta era inferior a $25 \mathrm{~mm}$, o risco de parto pré-termo antes de 32,35 e 37 semanas foi de $26,9,53,9$ e 73,1\%, respectivamente.

Kushnir et al. ${ }^{7}$ compararam medidas do colo uterino obtidas em gestações únicas e gemelares e concluíram que havia maior encurtamento do colo a partir da $20^{a}$ semana nas gestações gemelares. Isto explicaria a maior vulnerabilidade das gestações gemelares de evoluírem para trabalho de parto prematuro. Porém, o mecanismo envolvido no encurtamento do colo uterino não está estabelecido.

Portanto, a avaliação ultra-sonográfica transvaginal é capaz de quantificar com precisão a medida da cérvice e identificar os casos de colo curto. Estes estão associados a alto risco de parto pré-termo e poderiam se beneficiar de medidas específicas visando redução deste risco e do número efetivo de partos pré-termo.

$\mathrm{Na}$ tentativa de avaliar a influência da posição da paciente no comprimento do colo uterino, Arabin et al. ${ }^{8}$ compararam as medidas do colo uterino com as gestantes nas posições de decúbito e ortostática. Os autores observaram que as medidas obtidas na posição ortostática eram menores que as obtidas em decúbito, e a magnitude desta diferença aumentou com a idade gestacional.

A fim de estudar a influência da posição materna na medida da cérvice uterina nas gestações gemelares, realizamos o presente estudo.

\section{Pacientes e Métodos}

No período de maio de 1999 a julho de 2000, no Hospital das Clínicas da Faculdade de Medicina da Universidade de São Paulo, 50 gestações gemelares foram submetidas a avaliações ultrasonográficas com medida do colo uterino, nas posições em decúbito dorsal horizontal (DDH) e ortostática. O presente estudo foi aprovado pela Comissão de Ética em Pesquisa envolvendo Seres
Humanos (CAPPESQ) da Instituição acima referida e consentimento informado foi obtido de todas as participantes do estudo.

Foram excluídas as gestações gemelares com óbito de um dos fetos, malformações fetais, sindrome da transfusão feto-fetal, gêmeos coligados, feto acárdico, poliidrâmnio, amniorrexe ou que estivessem em trabalho de parto.

O grupo foi caracterizado em relação à idade materna e gestacional, paridade e raça. A corionicidade foi determinada durante os exames ultra-sonográficos examinando-se a presença ou ausência do sinal do "lambda", o número e a posição das placentas e determinando-se o gênero dos fetos; alternativamente, foi realizado exame anatomopatológico das placentas após o parto.

Os exames ultra-sonográficos do colo uterino foram iniciados na ocasião da primeira consulta pré-natal e repetidos em intervalos de 4 semanas. A avaliação da cérvice uterina foi realizada inicialmente com a paciente na posição de DDH e, após um período mínimo de 20 minutos em pé, o colo foi reavaliado na posição ortostática, com a paciente apoiando um dos pés sobre um degrau de $40 \mathrm{~cm}$.

Inicialmente, as avaliações foram realizadas com a paciente em posição ginecológica e com a bexiga vazia. $\mathrm{O}$ transdutor foi introduzido até o fórnice anterior da vagina; em um corte sagital do colo uterino foram identificados os orificios interno e externo, o canal e a mucosa endocervical. Ampliou-se a imagem de modo que o colo ocupasse $75 \%$ da tela e os "calipers" foram posicionados nos orifícios interno e externo. Três medidas foram obtidas durante intervalo de pelo menos 3 minutos e a menor medida foi registrada ${ }^{5}$.

Os resultados das gestações foram obtidos por meio de cartas-respostas, contato telefônico ou consulta de prontuário médico para determinar a idade gestacional do parto.

Para analisar a correlação entre as medidas do colo uterino obtidas na posição de decúbito e ortostática, e a correlação destas com a idade gestacional, foi empregada regressão linear simples. A diferença entre as medidas do colo uterino na posição ortostática e de DDH foi analisada com o teste pareado $t$ de Student. Essa diferença foi também expressa como percentual da medida obtida em decúbito $\{[$ (medida ortostática - medida decúbito)/medida decúbito] x 100\} para neutralizar o efeito da idade gestacional sobre a medida do colo uterino. A diferença percentual foi então analisada com teste $t$ de Student com amostra única comparando com média zero. 


\section{Resultados}

A idade média materna foi de 28,6 anos (desvio-padrão (DP) $=7,1$ anos). Onze $(22 \%)$ gestantes eram da raça branca, $38(76 \%)$ pardas e uma $(2 \%)$ negra. Quanto à paridade, 20 (40\%) eram primigestas e 19 (38\%) multiparas (dois ou mais partos anteriores). A corionicidade foi determinada em 43 casos $(86 \%)$, sendo oito gestações monocoriônicas diamnióticas (16\%) e 35 (70\%), dicoriônicas. A idade gestacional média do parto foi de 36,5 semanas ( $D P=2,6$ semanas).

A idade gestacional média na primeira avaliação foi de 23,7 semanas ( $\mathrm{DP}=6,4$ semanas). Nestes 50 exames, as medidas do colo uterino nas posições DDH e ortostática se correlacionaram inversamente com a idade gestacional $(\mathrm{DDH}$ : $r=-0,60 ; p<0,001$; ortostático: $r=-0,46 ; p=0,008$; Figura 1). A média da medida do colo uterino em $\mathrm{DDH}$ foi de $35,2 \mathrm{~mm}(\mathrm{DP}=9,9 \mathrm{~mm})$, e $33,4 \mathrm{~mm}$ $(\mathrm{DP}=9,5 \mathrm{~mm})$ na posição ortostática (média das diferenças ortostático vs $\mathrm{DDH}=-1,8 \mathrm{~mm} ; \mathrm{t}=1,96$; $\mathrm{p}=0,06)$. A diferença percentual não apresentou correlação significativa com a idade gestacional da avaliação $(r=0,26 ; p=0,07)$ e a média das diferenças percentuais foi de $-2,9 \%(t=1,0 ; p=0,3)$.

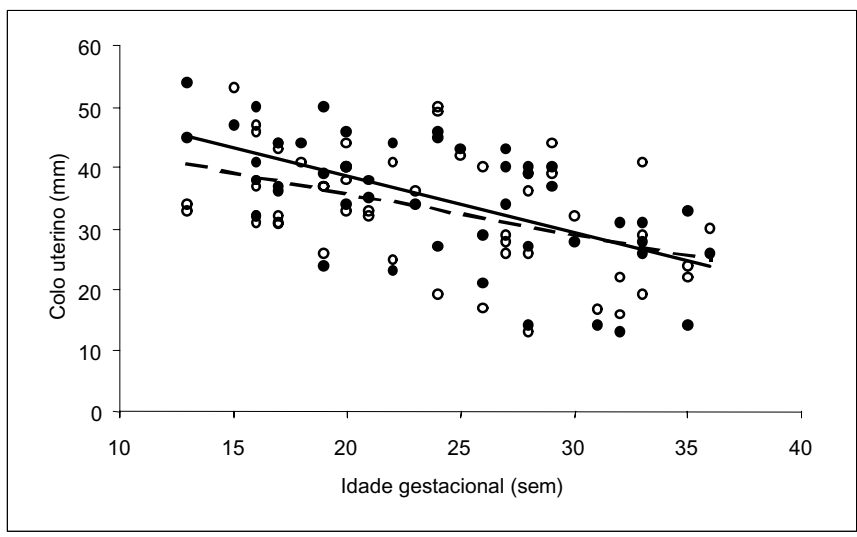

Figura 1 - Medidas do colo uterino durante a primeira avaliação ultra-sonográfica em 50 gestações gemelares nas posições de decúbito (círculos cheios) e ortostático (círculos abertos). Linha contínua: regressão linear das medidas na posição de decúbito em relação à idade gestacional; linha tracejada: regressão linear das medidas na posição ortostática em relação à idade gestacional.

Durante o seguimento subseqüente destas 50 gestações, foram realizadas 136 avaliações ultrasonográficas com medidas do colo uterino nas posições DDH e ortostática (média $=2,7$ avaliações por paciente; $\mathrm{DP}=1,4)$. A idade gestacional média das avaliações foi de 27 semanas ( $\mathrm{DP}=6,3$ semanas).

Considerando-se estas 136 avaliações, as medidas obtidas na posição DDH se correlacionaram com aquelas obtidas na posição ortostática $(\mathrm{r}=0,79 ; \mathrm{p}<0,001)$. As medidas do colo uterino nas posições DDH e ortostática correlacionaram-se inversamente com a idade gestacional (decúbito: $\mathrm{r}=-0,51 ; \mathrm{p}<0,0001$; ortostático: $\mathrm{r}=-0,49 ; \mathrm{p}<0,0001$; Figura 2). A média da medida do colo uterino em $\mathrm{DDH}$ foi de 33,5 mm (DP=10,8 $\mathrm{mm}$ ), e $31,8 \mathrm{~mm}$ $(\mathrm{DP}=9,6 \mathrm{~mm}$ ) na posição ortostática (média das diferenças ortostática vs $\mathrm{DDH}=-1,7 \mathrm{~mm} ; \mathrm{t}=3,01$; $\mathrm{p}=0,003)$. A diferença percentual não apresentou correlação significativa com a idade gestacional da avaliação $(r=0,14 ; p=0,10)$ e a média das diferenças percentuais foi de $-3,0 \%(t=-1,64 ; p=0,10)$.

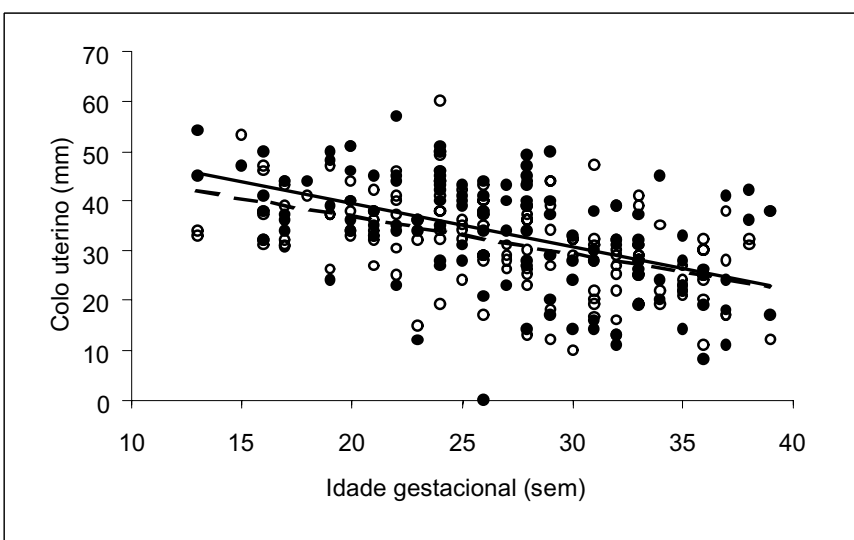

Figura 2 - Medidas do colo uterino em 136 avaliações ultra-sonográficas de 50 gestações gemelares nas posições de decúbito (círculos cheios) e ortostática (círculos abertos). Linha contínua: regressão linear das medidas na posição de decúbito em relação à idade gestacional; linha tracejada: regressão linear das medidas na posição ortostática em relação à idade gestacional.

\section{Discussão}

As altas taxas de morbiletalidade associadas às gestações gemelares se devem em grande parte à prematuridade. Cerca de 40 a $50 \%$ destas gestações evoluem para parto antes de 37 semanas, e as gestações gemelares respondem por cerca de $12 \%$ do total de nascimentos abaixo desta idade gestacional ${ }^{9,10}$.

A avaliação da medida do colo uterino pela ultra-sonografia transvaginal é o método diagnóstico que tem demonstrado melhor resultado na tentativa de predição do parto pré-termo, tanto nas gestações únicas como nas gemelares ${ }^{3,5,11}$. Skentou et al. ${ }^{12}$, em estudo envolvendo 464 gestações gemelares, avaliaram a medida do colo uterino na $23^{\text {a }}$ semana de gestação e concluíram que o risco para parto antes da $33^{a}$ semana aumentava conforme a medida do colo uterino diminuía. Quando o colo uterino media $60 \mathrm{~mm}$, o risco era de $2,5 \%$ e este aumentava gradativamente até $17 \%$ quando a medida cervical atingia $20 \mathrm{~mm}$; 
abaixo desta medida cervical, o risco aumentava exponencialmente e atingia $80 \%$ com $8 \mathrm{~mm}$.

No presente estudo a medida do colo uterino variou inversamente com a idade gestacional. Esses dados são semelhantes aos resultados encontrados por Ong et al. ${ }^{13}$, que avaliaram a evolução da medida do colo uterino em gestações gemelares na posição de decúbito dorsal horizontal. Arabin et al. ${ }^{8}$ também observaram que, com a evolução da gestação, as médias das medidas do colo uterino diminuíam de $50 \mathrm{~mm}$ a $27 \mathrm{~mm}$ na posição de decúbito dorsal horizontal e de $48 \mathrm{~mm}$ a $21 \mathrm{~mm}$ na posição ortostática.

Guzman et al. ${ }^{14,15}$, avaliando gestações únicas, na posição de decúbito, observaram que a medida do colo uterino era influenciada por pressão exercida sobre o fundo uterino. Portanto, a avaliação da medida cervical na posição ortostática demonstraria as mudanças sob pressão fisiológica.

O único estudo que comparou a diferença nas medidas do colo uterino nas posições de DDH e ortostática, em gestações gemelares, demonstrou diferença significativa nas medidas absolutas e percentuais, e esta diferença aumentou com o evoluir da gestação. No presente estudo, analisando-se as medidas obtidas em todas as avaliações, também foi observada diferença significativa. Entretanto, diferentemente dos resultados de Arabin et al. ${ }^{8}$, não observamos aumento da magnitude desta diferença com o evoluir da gestação. Isto pode ser devido à diferença entre os tamanhos amostrais dos dois estudos e à inclusão de três gestações trigemelares no estudo referido.

Esta diferença observada entre as medidas absolutas nas posições DDH e ortostática pode ser explicada pela mudança na orientação dos vetores de força nas diferentes posições maternas. Na posição de decúbito horizontal, ocorreria um alinhamento entre o eixo do canal cervical e o maior eixo longitudinal do corpo uterino, entretanto o efeito da força gravitacional seria menor. Já na posição ortostática, ocorreria um maior efeito da força gravitacional sobre o colo uterino, levando à diminuição de seu comprimento, apesar da diminuição simultânea do ângulo formado entre o canal cervical e o corpo uterino que, em tese, atenuaria o efeito da força gravitacional.

Ainda no presente estudo, quando a diferença entre as medidas obtidas em DDH e ortostática era expressa como porcentagem do valor obtido em decúbito, ela deixou de ser significativa. Acreditamos que a avaliação expressa em percentual seja melhor, uma vez que neutraliza o efeito da idade gestacional sobre a medida do colo uterino.

Pode-se concluir então que as avaliações do colo uterino com as pacientes na posição de DDH e ortostática fornecem informações similares.
Outros fatores de maior relevância devem estar envolvidos no processo fisiopatológico que determina o encurtamento do colo uterino nas gestações gemelares.

Uma vez que estas gestações de risco podem se beneficiar de condutas e orientações como o repouso, uso de tocolíticos, pesquisa ativa de infecção gênito-urinária materna e cerclagem uterina, o estudo de métodos diagnósticos para identificação de colos uterinos curtos é de fundamental importância.

\section{ABSTRACT}

Purpose: to compare cervical length measurements in twin pregnancies obtained by transvaginal ultrasound examination in the recumbent and standing positions.

Methods: fifty twin pregnancies underwent transvaginal ultrasound examinations to measure the cervical length with the women in recumbent and standing positions. The study was carried out between May 1999 and December 2000. The scans were repeated every 4 weeks and the total number of evaluations was 136. Two groups were analyzed: one included only the first ultrasound examinations carried out in each woman and the second group included all evaluations.

Results: in the first group, cervical length measurements in the standing and recumbent positions correlated inversely with the gestational age (recumbent: $r=-0.60 ; p<0.001$; standing: $r=-0.46 ; p=0.008)$. The mean measure in the recumbent position was $35.2 \mathrm{~mm}(\mathrm{SD}=9.9 \mathrm{~mm})$ and $33.4 \mathrm{~mm}$ $(S D=9.5 \mathrm{~mm})$ in the standing position. When the difference between the measure obtained in the standing and recumbent positions was expressed as percentage of the measure in the recumbent position, there was no significant association with gestational age $(p=0.07)$. When all evaluations were considered, there was a significant association between cervical length in the recumbent and standing positions $(r=0.79 ; p<0.001)$. The measures in recumbent and standing positions were inversely correlated with gestational age (recumbent: $p<0.0001$; standing: $p<0.0001$ ). The mean cervical length in the recumbent position was $33.5 \mathrm{~mm}$ $(S D=10.8 \mathrm{~mm})$ and $31.8 \mathrm{~mm}(S D=9.6 \mathrm{~mm})$ in the standing position. There was no significant association between cervical length difference expressed as percentage of the measure in the recumbent position and gestation.

Conclusion: cervical length measure obtained with the patients in the recumbent and standing positions provided similar information.

KEY WORDS: Preterm delivery. Twin pregnancy. Uterine cervix. Normal pregnancy. 


\section{Referências}

1. Michaels WH, Schreiber FR, Padgett RJ, Ager J, Pieper D. Ultrasound surveillance of the cervix in twin gestations: management of cervical incompetency. Obstet Gynecol 1991; 78:739-44.

2.Sperling L, Tabor A. Twin pregnancy: the role of ultrasound in management. Acta Obstet Gynecol Scand 2001; 80:287-99.

3. Roberts WE, Morrison JC. Has the use of home monitors, fetal fibronectin, and measurement of cervical length helped predict labor and/or prevent preterm delivery in twins? Clin Obstet Gynecol 1998; 41:95-102.

4. Crane JM, Van den Hof M, Armson BA, Liston R. Transvaginal ultrasound in the prediction of preterm delivery: singleton and twin gestations. Obstet Gynecol 1997; 90:357-63.

5. Iams JD, Goldenberg RL, Meis PJ, et al. The length of the cervix and the risk of spontaneous premature delivery. N Engl J Med 1996; 334:567-72.

6. Goldenberg RL, Iams JD, Miodovnik M, et al. The preterm prediction study: risk factors in twin gestations. Am J Obstet Gynecol 1996; 175:1047-53.

7. Kushnir O, Izquierdo LA, Smith JF, Blankstein J, Curet LB. Transvaginal sonographic measurement of cervical length. Evaluation of twin pregnancies. J Reprod Med 1995; 40:380-2.
8. Arabin B, Aardenburg R, van Eyck J. Maternal position and ultrasonic cervical assessment in multiple pregnancy. Preliminary observations. J Reprod Med 1997; 42:719-24.

9. Ho SK, Wu PY. Perinatal factors and neonatal morbidity in twin pregnancies. Am J Obstet Gynecol $1975 ; 122: 979-87$.

10.Gardner MO, Goldenberg RL, Cliver SP, Tucker JM, Nelson KG, Copper RL. The origin and outcome of preterm twin pregnancies. Obstet Gynecol 1995; 85:553-7.

11. Chuileannáin FN, Brennecke S. Prediction of preterm labour in multiple pregnancies. Baillieres Clin Obstet Gynecol 1998; 12:53-66.

12. Skentou C, Souka AP, To MS, Liao AW, Nicolaides $\mathrm{KH}$. Prediction of preterm delivery in twins by cervical assessment at 23 weeks. Ultrasound Obstet Gynecol 2001; 17:7-10.

13. Ong S, Smith A, Smith N, Campbell D, Wilson A. Cervical length assessment in twin pregnancies using transvaginal ultrasound. Acta Obstet Gynecol Scand 2000; 79:851-3.

14.Guzman ER, Houlihan C, Vintzielos A. Sonography and transfundal pressure in the evaluation of the cervix during pregnancy. Obstet Gynecol Surv 1995; 50:395-403.

15.Guzman ER, Rosenberg JC, Houliban C, Ivan J, Waldron R, Knuppel R. A new method using vaginal ultrasound and transfundal pressure to evaluate the asymptomatic incompetent cervix. Obstet Gynecol 1994; 83:248-52.

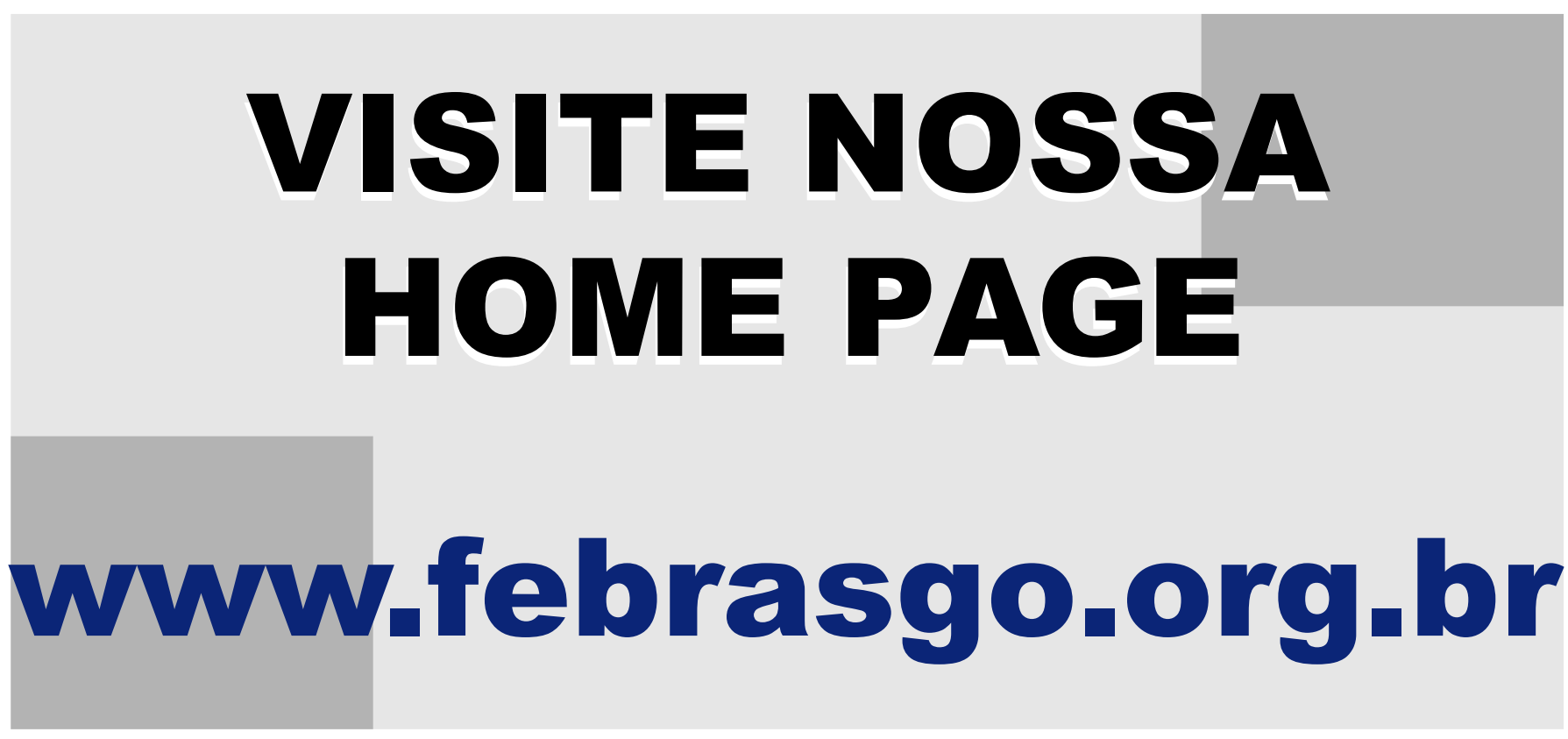

